Trauma Berufskrankh 2004 - 6 [Suppl 4] : S446-S449 DOI 10.1007/s10039-003-0789-x

Online publiziert: 22.0ktober 2003

(c) Springer-Verlag 2003

H.-J.Wirthl

Landesverband Hessen-Mittelrhein und Thüringen der gewerblichen Berufsgenossenschaften, Mainz

\title{
Organisation der Heil- und Hilfsmittelversorgung in der gesetzlichen Unfallversicherung
}

ersatzstücke, orthopädische und andere Hilfsmittel ( $\$ 31$ Abs. 1 SGB VII). Ziel der Ausstattung mit Hilfsmitteln ist es, die Folgen von Gesundheitsschäden zu mildern oder auszugleichen und so den Erfolg der Heilbehandlung insgesamt zu sichern.

Körperersatzstücke ersetzen verloren gegangene oder funktionsunfähige Körperteile. Orthopädische Hilfsmittel dienen der Sicherstellung des Behandlungserfolgs im Bereich des Stütz- und Bewegungsapparats. Im Sinn einer umfassenden Betrachtung sind jedoch grundsätzlich alle Gegenstände, die dem Ausgleich von Defiziten auch anderer Körperfunktionen und an Sinnesorganen dienen (z. B. Seh- und Hörhilfen, Orientierungshilfen für Blinde, Krankenfahrstühle, Hilfen zum Lesen und Sprechen) als Hilfsmittel anzusehen. Die Hilfsmittelversorgung umfasst auch die notwendige Änderung, Instandsetzung und Ersatzbeschaffung einschließlich der Ausbildung im Gebrauch der Hilfsmittel. Mindestgebrauchszeiten sind nicht vorgesehen.

\section{Rechtlicher Rahmen}

Der Gesetzgeber hat im SGB VII lediglich den Grundanspruch der Versicherten auf eine sachgerechte Ausstattung mit Hilfsmitteln geregelt. Darüber hinaus hat er die Bundesregierung ermächtigt, durch Rechtsverordnung mit Zustimmung des Bundesrats die Voraussetzungen sowie den Art und den Umfang der Ausstattung mit Hilfsmitteln im Einzelfall zu regeln. Die in diesem Zusammenhang ergangene Verordnung über die orthopädische Versorgung Unfallverletzter vom 18.7.1973 gilt weiter. Der Verordnungsgeber hat jedoch ganz bewusst davon abgesehen, Einzelheiten zu regeln und überließ seine Regelungsbefugnis den Unfallversicherungsträgern. Diese haben entsprechend ihrer gesetzlich eingeräumten Befugnis in $\$ 31$ Abs. 2 Satz 2 SGB VII "Gemeinsame Richtlinien der Unfallversicherungsträger über Hilfsmittel (UV-Hilfsmittelrichtlinien)“ erlassen. Diese Richtlinien sind wiederum so flexibel gestaltet, dass im Einzelfall stets angemessene Hilfe geleistet werden kann, um der weitgehenden Verpflichtung der gesetzlichen Unfallversicherung zur Heilbehandlung mit allen geeigneten Mitteln stets gerecht werden zu können. Die Richtlinien entfalten den Charakter verbindlicher Rechtsnormen, sodass sie eine Selbstbindung der UVTräger bewirken. Sie regeln allerdings lediglich die Art und den Umfang der Versorgung. Zugunsten eines Versicherten kann hiervon unter Berücksichtigung der Umstände des Einzelfalls im Sinn eines Auswahlermessens abgewichen werden.

\section{Was sind Hilfsmittel?}

Als Hilfsmittel kommen nach den UVHilfsmittelrichtlinien insbesondere in Frage: 
- Kunstglieder, Kunstaugen, Zahnersatz und andere künstliche Körperteile

- Stützapparate, orthopädisches Schuhwerk, Stockstützen und andere Gehilfen

- Rollstühle, Kraftfahrzeuge

- Geräte zur Unterstützung oder zum Ersatz von Körperfunktionen

- Perücken

- technische Arbeitshilfen, Zubehör zu Hilfsmitteln

- Blindenführhunde

Im Unterschied $\mathrm{zu}$ anderen Sozialleistungsbereichen können auch allgemeine Gebrauchsgegenstände des täglichen Lebens die Voraussetzungen des Hilfsmittelbegriffs erfüllen. Im Prinzip kann jeder Gegenstand, der wegen der Art der Gesundheitsstörung einer besonderen Ausgestaltung bedarf, als Hilfsmittel im Sinn von $\$_{31}$ SGB VII angesehen werden. Der Unfallversicherungsträger hat dann die Mehrkosten der Anpassung zu tragen.

\section{Festbeträge}

Für die Versicherten der gesetzlichen Unfallversicherung werden Hilfsmittel grundsätzlich kostenfrei zur Verfügung gestellt. So weit allerdings im Bereich der gesetzlichen Krankenversicherung Festbeträge festgesetzt sind, gelten diese auch in der gesetzlichen Unfallversicherung (\$31 Abs. 1 Satz 3 SGB VII). Die Festbeträge werden von den gesetzlichen Krankenversicherungsträgern auf Länderebene festgesetzt und gelten für Versicherte der gesetzlichen Unfallversicherung unmittelbar. Der UV-Träger übernimmt die Kosten des Hilfsmittels in Höhe dieser Beträge. Verordnet der Arzt ein Hilfsmittel, dessen Preis den Festbetrag überschreitet, muss er den Versicherten auf die von diesem zu übernehmenden Mehrkosten hinweisen. Eine Ausnahme gilt lediglich dann, wenn das Ziel der Heilbehandlung durch Festbetragsmittel nicht erreicht werden kann. Dann ist eine Begrenzung ausgeschlossen. Bei entsprechender medizinischer Begründung hat der UV-Träger die Mehrkosten zu übernehmen. Auch dies ist Ausdruck des umfassenden Rehabilitationsauftrags in der gesetzlichen Unfallversicherung.

Trauma Berufskrankh 2004 - 6 [Suppl 4] : S446-S449

DOI 10.1007/s10039-003-0789-x

C Springer-Verlag 2003

\section{H.-J.Wirthl}

\section{Organisation der Heil- und Hilfsmittelversorgung in der gesetzlichen Unfallversicherung}

\section{Zusammenfassung}

Die Heilbehandlung in der gesetzlichen Unfallversicherung umfasst auch die Versorgung mit Heil- und Hilfsmitteln. Damit sollen der Erfolg der Heilbehandlung gesichert und die Folgen von Gesundheitsschäden körperlicher, seelischer oder geistiger Natur gemildert oder ausgeglichen werden. Dies ist Bestandteil des gesetzlichen Auftrags, die Gesundheit und die Leistungsfähigkeit der Versicherten nach Eintritt von Arbeitsunfällen oder Berufskrankheiten mit allen geeigneten Mitteln wieder herzustellen. Vor dem Hintergrund der eklatanten Kostensteigerung insbesondere im Bereich der Hilfsmittelversorgung erscheint es zulässig und geboten, neue Strategien zu entwickeln. Bei der Ausübung des den Trägern der Unfallversicherung eingeräumten Ermessens ist im Einzelfall jedoch stets der Zweck der Ermächtigung zu beachten. Die Ausstattung mit Hilfsmitteln muss sich auch an den individuellen Bedürfnissen der Versicherten orientieren und hohen Qualitätsansprüchen genügen. Wichtig sind einheitliche Regelungen für möglichst alle UV-Träger. Angesichts der Komplexität des Themas beschränkt sich der Beitrag im Wesentlichen auf den Bereich der Hilfsmittelversorgung.

\section{Schlüsselwörter}

Rehabilitation · Heilbehandlung · Hilfsmittel . Heilmittel $\cdot$ Wirtschaftlichkeit und Sparsamkeit

\section{Organising the provision of medicines and aids within the scope of statutory accident insurance}

\section{Abstract}

Curative treatment under statutory accident insurance also encompasses the provision of medicinal products and medical aids. The intention is to ensure that the curative treatment is successful and to mitigate or compensate for the effects of physical, mental or psychological injury. This is part of our statutory mandate, which is to apply all appropriate means to restore insured persons' health and fitness after occupational accidents or work-related disorders. In view of the cost explosion, especially in the provision of medical aids, the develpment of new strategies seems both permissible and necessary. However, when exercising the discretion they are entitled to use, accident insurance institutions must always bear in mind the purpose of this entitlement in each case. The provision of medical aids must be centred on the individual needs of the insured person and meet exacting quality standards. Uniform regulations should apply to all accident insurance institutions if possible. Given the complexity of this subject, the paper is essentially restricted to the provision of medical aids.

\section{Keywords}

Rehabilitation · Curative treatment · Medical aids Medicinal preparations · Efficiency and economy 
Bei Hilfsmitteln, für die keine Festbeträge vorgesehen sind, werden die Preise in Verhandlungen zwischen überregionalen Verbänden der Anbieter und den Landesverbänden der Krankenkassen festgelegt. Sie handeln Preisobergrenzen aus. Dies trägt einerseits zur Einkommenssicherung auch kleinerer Anbieter bei. Andererseits soll dadurch die flächendeckende Versorgung der Versicherten selbst in entlegendsten Gebieten sichergestellt werden. Insbesondere bei denjenigen Anbietern, die z. B. über höhere Kundenfrequenzen und effizientere Betriebsabläufe verfügen oder auf günstigere Einkaufskonditionen zurückgreifen können, sollen jedoch Preisspielräume nach unten im Sinn des gegenseitigen Wettbewerbs genutzt werden.

\section{Eigenanteil der Versicherten}

In bestimmten Fällen hat der Versicherte auch in der gesetzlichen Unfallversicherung einen Eigenanteil zu übernehmen. Dies kann z. B. bei der Versorgung mit Prothesenschuhen oder orthopädischen Schuhen der Fall sein. Hier wird für den nicht verletzten Fuß bei der Erstausstattung stets ein Schuh kostenfrei mitgeliefert. Bei der Ersatzlieferung fällt für den nicht verletzten Fuß ein Eigenanteil an, der sich nach den in der Kriegsopferversorgung maßgebenden Sätzen richtet. Auch die Kosten für die infolge gewöhnlicher Abnutzung erforderliche Besohlung sind vom Versicherten selbst zu tragen.

\section{Kostenentwicklung}

Auch die gesetzliche Unfallversicherung bleibt von Kostensteigerungen nicht verschont. Die Aufwendungen für ambulante Heilbehandlung im Bereich der gewerblichen Berufsgenossenschaften stiegen von 507 Mio. EUR 1994 um 34\% auf 679 Mio. EUR 2002 (• Abb. 1). Hochrechnungen aus Stichproben ergaben, dass die in diesen Beträgen enthaltenen Aufwendungen für Hilfsmittel mit einer Steigerung um $78 \%$ von 79 Mio. EUR 1994 auf 141 Mio. EUR 2002 förmlich explodiert sind (• Abb. 1). Während der Anteil der Hilfsmittel an den Aufwendungen für ambulante Heilbehandlung 1994 noch 15,6\% betrug, waren es 2002 bereits $20,7 \%$. Der zunehmende Trend ist ungebrochen.

\section{Spannungsfeld "Wirtschaftlichkeit und Sparsamkeit"?}

Der im Bereich der Sozialversicherung also auch der gesetzlichen Unfallversicherung - gültige Grundsatz der Wirtschaftlichkeit und Sparsamkeit steht nicht im Widerspruch zum Grundsatz der Heilbehandlung mit allen geeigneten Mitteln. \$8 Abs. 1 des Vertrags Ärzte-Unfallversicherungsträger verpflichtet den Arzt, bei der ärztlichen Behandlung das Gebot der Wirtschaftlichkeit zu erfüllen. Außer einer evtl. vorhandenen persönlichen Motivation existiert jedoch für Ärzte und Versicherte gleichermaßen kein effektiver Anreiz zu Kosten sparendem Verhalten. In der gesetzlichen Unfallversicherung gibt es für Ärzte keine Ausgabenbegrenzung wie in der Krankenversicherung, und für Versicherte sind Hilfsmittel in der Regel unabhängig vom ausgewählten Anbieter kostenfrei zu beziehen. Bewährte Strukturen zu verlassen, ist zudem unbequem. Ein wirksamer Wettbewerb unter den Anbietern wird auf diese Weise nicht zu erzielen sein.

Der Vertrag Ärzte-UV-Träger regelt im Sinn der Qualitätssicherung, dass nur der D- oder H-Arzt (Ausnahme Seh- und Hörhilfen) Hilfsmittel verordnen kann. Hierzu verwendet er das Muster 16 aus der vertragsärztlichen Versorgung. Darüber, wem der Arzt seine Verordnung zu übergeben hat, existiert im Ärztevertrag keine spezifische Regelung. In aller Regel wird der

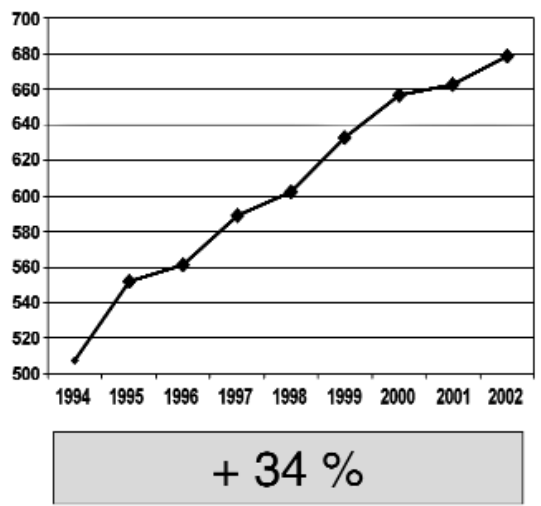

Abb. $1 \Delta$ Ausgaben in Mio. EUR von 1994-2002, links ambulante Heilbehandlung (insgesamt), rechts davon Hilfsmittel (Hochrechnung nach Stichprobe)

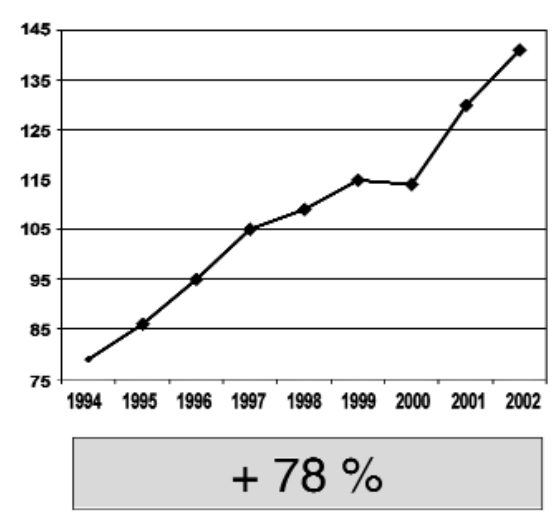

Arzt die Verordnung dem Versicherten selbst übergeben, der sich anschließend das Hilfsmittel besorgt und den Anbieter hierzu auswählt.

Allerdings hat der Gesetzgeber den UV-Trägern in \$26 Abs. 5 SGB VII die Kompetenz zugewiesen, im Einzelfall die Art, den Umfang und die Durchführung der Heilbehandlung und der Leistungen zur Teilhabe sowie die Einrichtungen, die diese Leistungen erbringen, nach pflichtgemäßem Ermessen zu bestimmen. Die Vorschrift regelt die umfassende Zuständigkeit und Gesamtverantwortung des UV-Trägers für die Heilbehandlung und Leistungserbringung. In der Konsequenz bedeutet dies, dass der Versicherte zwar einen Grundanspruch, jedoch keinen Rechtsanspruch auf ein bestimmtes Hilfsmittel hat. Über das konkrete Hilfsmittel entscheidet der UV-Träger im Rahmen seines Auswahlermessens. Auch das Wahlrecht des Leistungsanbieters steht im Gegensatz zur Krankenversicherung ausschließlich dem UV-Träger zu. Sämtliche Leistungen können in völliger Vertragsfreiheit auf dem Markt nachgefragt werden. Der UV-Träger ist auch nicht verpflichtet, die Vielfalt der Leistungserbringer oder gar deren Globalität zu berücksichtigen. Dies ergibt sich auch aus dem insgesamt gesehen verhältnismäßig geringen Marktanteil der UV-Träger.

Das Gericht Erster Instanz beim EUGH entschied mit Urteil vom 4.3.2003, dass das Beschaffungsverhalten von Sozialversicherungsträgern, z. B. beim Einkauf von Hilfsmitteln oder medizinischen Leistungen, keine wirtschaftliche unterneh- 
merische Aktivität darstellt. Die Entscheidung stützt sich darauf, dass die Weiterverwendung der erworbenen Güter zu nicht wirtschaftlichen, sondern rein sozialen Zwecken zu berücksichtigen ist. Kauft ein Sozialversicherungsträger deshalb z. B. in großen Mengen Güter oder Dienstleistungen ein, um sie im Rahmen einer nicht wirtschaftlichen (sozialen) Tätigkeit zu verwenden, kann das Einkaufsverhalten nicht vom späteren Verwendungszweck losgelöst betrachtet werden und ist als nicht unternehmerisch einzustufen. Dies bedeutet, dass das Nachfrageverhalten von Sozialversicherungsträgern sich nicht an den Vorgaben des europäischen Wettbewerbsrechts (Vorschriften zu Ausschreibungen, Verbot des Missbrauchs einer Markt beherrschenden Stellung) ausrichten muss. Es bleibt abzuwarten, ob der Europäische Gerichtshof in anderen Verfahren die Rechtsprechung des Gerichts Erster Instanz bestätigt.

\section{Berufsordnung der Ärzte}

Bei einer entsprechenden Aufforderung des UV-Trägers verstoßen Ärzte nicht gegen die Berufsordnung, denn darin ist ein hinreichender Grund für den Verweis des Patienten an bestimmte Leistungserbringer im Sinn der Berufsordnung zu sehen.

\section{Neue Strategien}

Mit dem Ziel einer Ausgabenbegrenzung durch Förderung des Wettbewerbs unter den Anbietern sind neue Wege bei der Hilfsmittelversorgung zulässig. Denkbar sind z. B.:

- Miete oder Wiedereinsatz gebrauchter Hilfsmittel und die Bildung von Hilfsmittelpools,

- Auswahl bestimmter Leistungsanbieter,

- Erstellung von Indikationslisten zur Versorgung bei definierten Verletzungen und Diagnosen sowie u. U. auch

- eigene Festbetragsregelungen für die gesetzliche Unfallversicherung.

\section{Orientierungsmaßstäbe}

Bei der Ausübung des Ermessens im Einzelfall ist stets der Zweck der Ermächtigung zu beachten. Maßgebend ist insbesondere der Grundsatz der optimalen Rehabilitation mit allen geeigneten Mitteln. Eine Billigversorgung ist abzulehnen. Höchstmögliche Versorgungsqualität bildet den Maßstab insbesondere bei individuell anzupassenden Hilfsmitteln, bei denen eine enge Kooperation zwischen Arzt, Orthopädietechniker und Versichertem erforderlich ist. Dabei sind insbesondere die Art und die Schwere der Verletzungsfolgen, die örtlichen, familiären sowie beruflichen Verhältnisse und auch angemessene Wünsche des Versicherten neben der grundsätzlichen Geeignetheit eines Hilfsmittels zu berücksichtigen. Gerade im Rahmen der Erstoder Akutversorgung darf keine Verzögerung bei der Hilfsmittelausstattung eintreten. Besonders bedeutsam im Hinblick auf die Wirksamkeit und Akzeptanz sowohl bei den Hilfsmittelanbietern als auch der Ärzteschaft ist, dass einheitliche und möglichst für alle UV-Träger gültige Regelungen gefunden werden.

\section{Fazit}

Die überproportionalen Kostensteigerungen der letzten Jahre zeigen, dass die Unfallversicherungsträger ihre Anstrengungen im Bereich der Hilfsmittelversorgung verstärkt auf wirtschaftliche Aspekte richten müssen. Es sollte möglich sein, hierzu wirksame Strategien zu entwickeln und bei den Anbietern zweifellos vorhandene Preisspielräume zu nutzen, ohne den Grundsatz der Rehabilitation mit allen geeigneten Mitteln zu verlassen. Eine Vielzahl von Einzelregelungen würde jedoch eher zur Verwirrung unter der Ärzteschaft und den Anbietern führen. Für den Erfolg der Maßnahmen wird es deshalb auch wesentlich auf eine möglichst einheitliche Vorgehensweise der UV-Träger ankommen.

\section{Korrespondierender Autor H.-J. Wirthl}

Landesverband Hessen-Mittelrhein und Thüringen der gewerblichen Berufsgenossenschaften, Wilhelm-Theodor-Römheld-Straße 15,55130 Mainz, Tel.: 06131-802226, Fax: 06131-802191,

E-mail:service@mainz.lvbg.de 